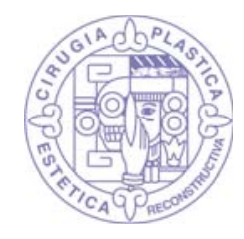

\title{
Manejo del paciente pediátrico quemado mediante la escalera reconstructiva
}

\author{
Handling of the pediatric burned patient through the reconstructive ladder \\ Dr. Patricio Canepa Fernández,* Dra. María Zulema Cantú Cantú,** \\ Dr. Joel Francisco Márquez Medina, ${ }^{* * *}$ Dr. Luis César Valencia García,* \\ Dr. Kist Aguilar Christian****
}

Palabras clave: Paciente pediátrico quemado, escalera reconstructiva.

Key words: Pediatric burned patient, reconstructive ladder.

\section{RESUMEN}

Los pacientes quemados representan un gran reto para los cirujanos plásticos; el espectro de las lesiones va desde quemaduras de primer grado, hasta quemaduras de tercer grado con exposición de estructuras nobles, siendo en ocasiones necesario el tratamiento radical. Es necesario que el cirujano plástico tenga un amplio repertorio de opciones para el manejo del paciente quemado. Presentamos cuatro casos de pacientes con diferentes grados de quemaduras, los cuales fueron manejados con base en la escalera reconstructiva, presentando buenos resultados tanto funcionales como estéticos. Concluimos que la escalera reconstructiva es el principio en el que se debe basar la cirugía reconstructiva, particularmente para el manejo del paciente quemado, por eso el cirujano plástico debe tener un amplio conocimiento de las opciones para resolver un problema y valorar los riesgos contra los beneficios, para ofrecer el mejor tratamiento para el paciente quemado.

\section{ABSTRACT}

Burned patients represent a great challenge for plastic surgeons, the spectrum of injuries ranging from first degree burns, to third degree burns with exposure of noble structures, sometimes requiring radical management. It is necessary that the plastic surgeon have a wide repertoire of options for the reconstruction of the burned patient. We present 4 cases of patients with different degrees of burns, which were managed based on the reconstructive ladder, presenting good results both functional and aesthetic. We concluded that the reconstructive ladder is the principle on which reconstructive surgery should be based, particularly for the management of the burned patient, so the plastic surgeon must have a wide knowledge of the options to solve a problem and assess the risks against the benefits, to offer the best treatment for the burned patient.

\footnotetext{
* Médico residente de cirugía plástica y reconstructiva. Hospital General «Dr. Rubén Leñero».

** Cirujano Plástico adscrito a la Unidad de Quemados. Hospital Pediátrico de Tacubaya. *** Plástico adscrito a la Unidad de Quemados del Hospital Pediátrico de Tacubaya.

**** Cirujano general. Hospital General «Dr. Fernando Quiroz Gutiérrez».

Los autores de este artículo no tienen conflicto de intereses qué declarar.

Recibido: 05 julio 2018

Aceptado para publicar: 01 agosto 2018
}

\section{INTRODUCCIÓN}

E I manejo del paciente quemado representa un reto para el cirujano plástico. El espectro de las lesiones va desde quemaduras de primer grado hasta quemaduras de tercer grado con exposición de estructuras nobles, o quemaduras que requieren manejo radical mediante la amputación de alguna extremidad, con secuelas devastadoras tanto funcionales como estéticas. Por tal motivo, el cirujano plástico debe tener un amplio arsenal de herramientas para tratar este tipo de lesiones.

Existen múltiples técnicas para el cierre de heridas que están disponibles para el cirujano reconstructivo. La lógica dicta que la opción de cierre debe comenzar con el método más simple, mientras que el cirujano debe tener en cuenta que en determinadas circunstancias se requieren técnicas más sofisticadas para obtener un mejor resultado.

La escalera reconstructiva es el espectro de opciones de cierre disponibles para las heridas y en la mente del cirujano reconstructivo se debe lograr el cierre con la técnica efectiva más simple.

El objetivo de este trabajo es presentar cuatro casos representativos de pacientes pediátricos quemados que se manejaron mediante los principios de la escalera reconstructiva.

\section{CASOS CLÍNICOS}

Caso 1. Niño de 18 meses de edad con quemaduras por escaldadura de segundo grado superficial y profundo. En la región inguinal 
persiste área cruenta, por lo que se decidió realizar resección de la misma con cierre directo presentando mejores resultados estéticos y funcionales (debido al trazado de la resección) que si hubiera sido manejado con un injerto cutáneo (Figuras 1 A y B).

Caso 2. Niña de siete años de edad con quemaduras por corriente eléctrica de tercer grado en la comisura labial derecha por morder un cable de cargador de celular. Una vez delimitada la lesión, se procedió a realizar un colgajo de avance en $\mathrm{V}-\mathrm{Y}$ de mucosa oral para cubrir el defecto, evitando así la microstomía, con secuelas estéticas y funcionales mínimas (Figuras 2 A-D).

Caso 3. Niño de 12 años con quemaduras por corriente eléctrica que ameritaron amputación tipo Chopart en miembro inferior izquierdo, dejando un área cruenta en el tercio inferior de la pierna con exposición de la tibia. Se realizó un colgajo sural reverso cruzado y se liberó a los 21 días permitiendo deambular al mes (Figuras 3 A-D).

Caso 4. Niño de 14 años con quemaduras por electricidad que tenía área cruenta en el pie izquierdo con exposición de primero y segundo metatarsianos. Se manejó mediante un colgajo radial libre con excelentes resultados cosméticos y funcionales (Figuras 4 A-D).

\section{RESULTADOS}

En los cuatro casos presentados se obtuvieron resultados adecuados tanto en lo funcional como en lo estético. En el primer caso se evitó

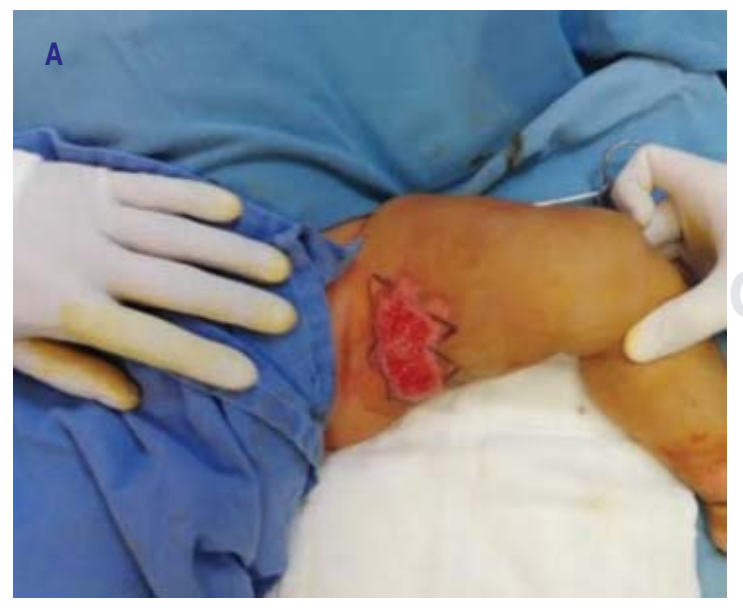

la toma de un injerto cutáneo que, además de la morbilidad, dejaría secuelas estéticas debido a la pigmentación. En el segundo caso se realizó un colgajo de mucosa para evitar la formación de cicatrices en la región de la comisura, evitando así la necesidad de hacer un colgajo de espesor total que dejaría secuelas estéticas y funcionales importantes. El tercer caso se resolvió mediante un colgajo a distancia, debido a que no se contaba con recurso microquirúrgico en ese momento, evitando así una amputación a un nivel más proximal. El cuarto caso se resolvió por medio de un colgajo libre, que, debido a las características de la zona donadora, dio un excelente resultado estético; asimismo, la zona donadora tiene poca morbilidad si se lleva a cabo la técnica de manera adecuada.

\section{DISCUSIÓN}

Los pacientes quemados siempre han representado un gran reto para los cirujanos plásticos. Las lesiones pueden ser desde algo simple a casos muy complejos que requieren un conocimiento profundo de la cirugía plástica y en muchas ocasiones es necesario utilizar diversos manejos para un mismo paciente. Los pacientes que sufren quemaduras con frecuencia presentan secuelas psicológicas, por esta razón muchos pacientes desarrollan una aversión a continuar con el tratamiento médico. Por tal motivo es importante seleccionar la mejor operación con la mayor probabilidad de éxito, la menor morbilidad postquirúrgica y un periodo de rehabilitación

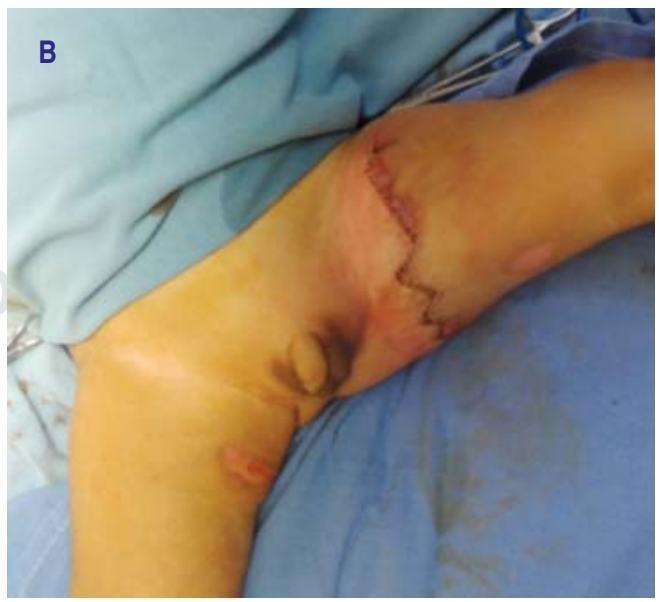


corto que involucre un mínimo de procedimientos quirúrgicos y éstos sean entendibles para el paciente. ${ }^{1}$ De esta manera, la escalera reconstructiva plantea al cirujano plástico diferentes alternativas terapéuticas para la toma de decisiones, desde lo más simple a lo más complejo para que en caso de fallo permita ir pasando por etapas más seguras. ${ }^{2}$

\section{Figuras 2.}

A) Quemadura en la comisura labial derecha. B) Marcado del colgajo de avance en $\mathrm{V}$-Y de mucosa oral. C) Disección del colgajo. D) Resultado a las dos semanas de la cirugía.
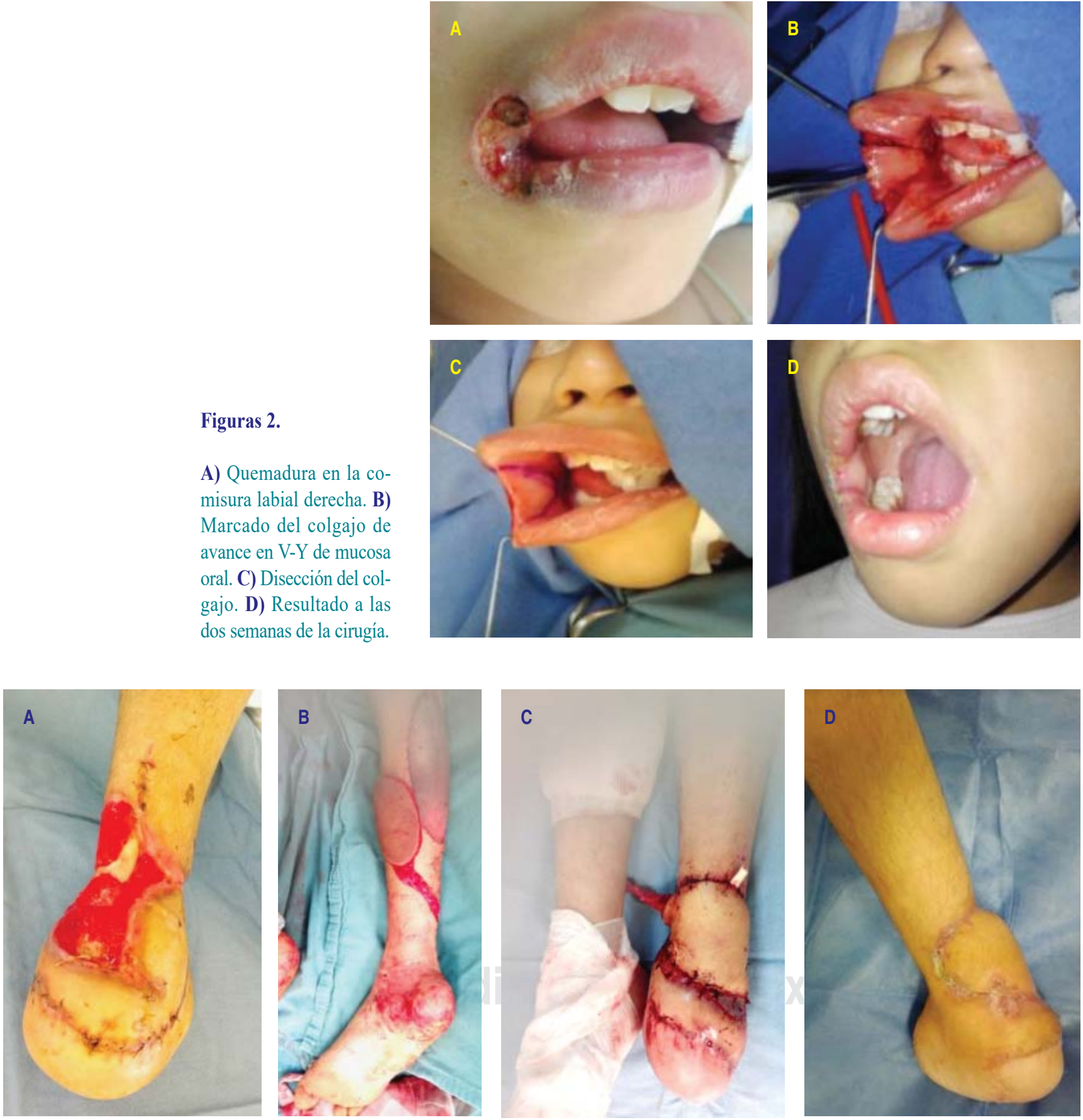

Figuras 3. A) Defecto en el tercio inferior de la pierna con exposición de la tibia. B) Disección del colgajo sural reverso. C) Postquirúrgico inmediato. D) Postquirúrgico tardío. 

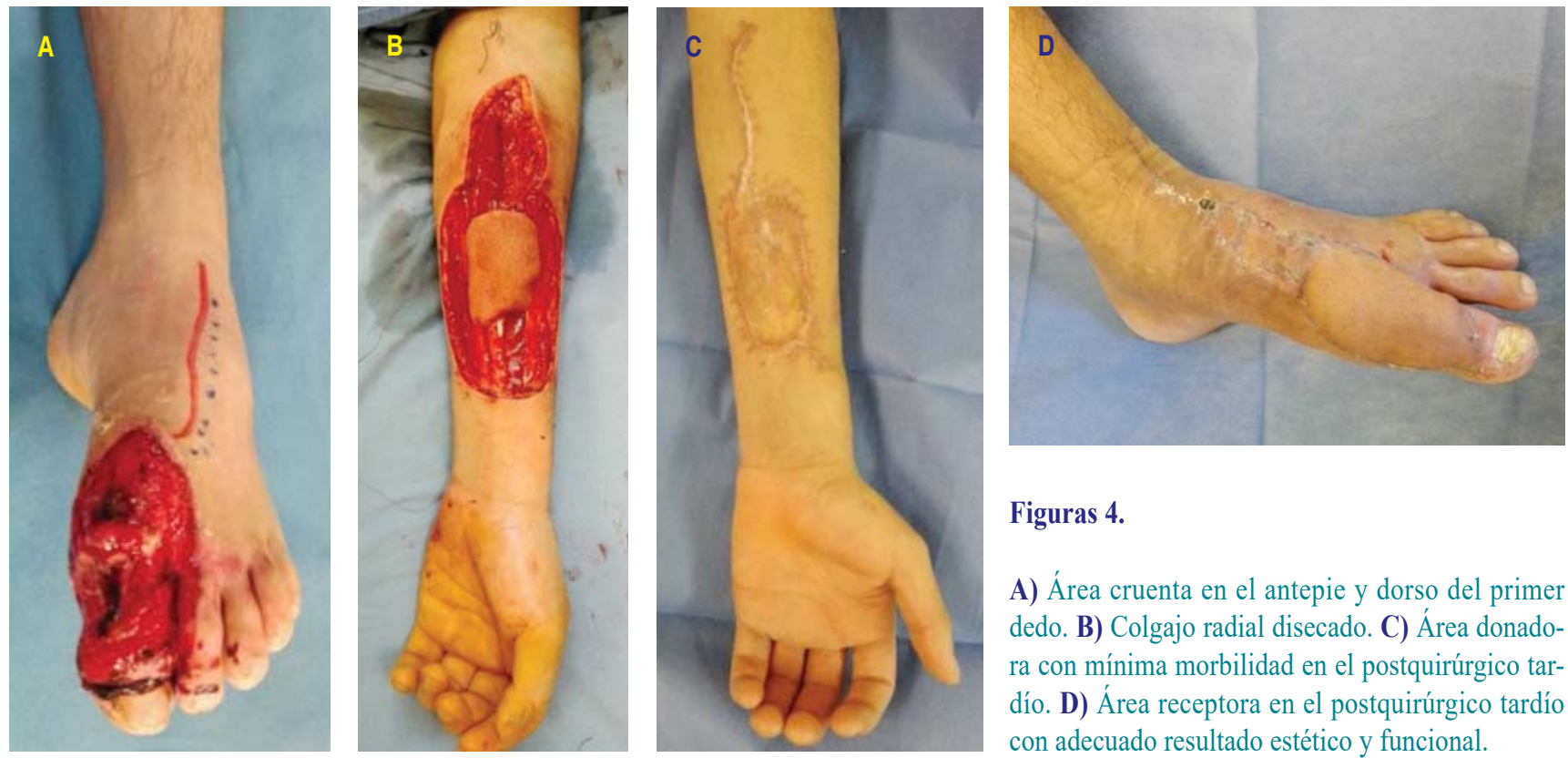

\section{Figuras 4.}

A) Área cruenta en el antepie y dorso del primer dedo. B) Colgajo radial disecado. C) Área donadora con mínima morbilidad en el postquirúrgico tardío. D) Área receptora en el postquirúrgico tardío con adecuado resultado estético y funcional.

La escalera reconstructiva inicia con el cierre por segunda intención, seguido por el cierre primario, como en el primer paciente; el injerto cutáneo, la expansión tisular, colgajos locales y a distancia como en los casos 2 y 3, o los colgajos libres como el colgajo radial libre realizado al paciente del caso 4; estos últimos fueron los más complejos. De esta manera, tenemos un amplio repertorio de opciones terapéuticas que deben ser individualizadas para cada paciente con la finalidad de obtener los mejores resultados estético-funcionales con las menores complicaciones posibles. ${ }^{3}$

En las últimas décadas se ha descrito el manejo con colgajos libres para pacientes quemados, en un principio para el tratamiento de las secuelas, pero con el tiempo se integraron como una técnica más en el manejo. Su indicación es más común en la cobertura de tejidos nobles (hueso, articulaciones, tendones, nervios y estructuras vasculares). ${ }^{4}$ En el caso de los colgajos libres, el tiempo para la reconstrucción se ha clasificado en dos categorías mayores: reconstrucción primaria, que comprende los subgrupos: inmediata (menos de cinco días), temprana (dentro de los cinco y 21 días) e intermedia (dentro de los 21 días a las seis semanas); la reconstrucción secundaria o tardía incluye todos los colgajos realizados después de las seis semanas de la lesión, ${ }^{5}$ aunque todavía hay estudios que discuten cuándo es el tiempo adecuado para la reconstrucción, ya que se ha visto que el índice de complicaciones aumenta entre los días 5 y 21 después de la lesión. 6

En los pacientes que sufren quemaduras o trauma de los labios debemos tener en cuenta dos objetivos: alcanzar un buen resultado estético y conservar la función labial, ya que esto conlleva una adecuada expresión facial, un involucramiento en la mímica facial, así como de la identidad de la persona, sin dejar a un lado la competencia oral. La primera causa de lesiones labiales es de origen carcinógeno, que en el $90 \%$ de los casos abarca el labio inferior, seguida por trauma, quemaduras y malformaciones. ${ }^{7}$ Los aspectos importantes que se deben considerar en la valoración y reconstrucción labial son la profundidad del defecto, localización, proporción del volumen perdido y el compromiso de la comisura. ${ }^{8}$ En nuestro caso 2 se decidió un colgajo de avance $\mathrm{V}-\mathrm{Y}$, ideal para defectos pequeños.

Los casos presentados demuestran que el manejo del paciente quemado implica un gran reto para el cirujano plástico, que necesita un dominio anatómico y un amplio conocimiento para poder tratar esta compleja patología. 


\section{CONCLUSIONES}

El paciente quemado presenta un espectro de lesiones que requieren un dominio de la cirugía plástica. El manejo de éstas va desde el cierre por segunda intención hasta la utilización de colgajos microquirúrgicos, por tal motivo el cirujano plástico debe tener un amplio conocimiento y un criterio acertado para poder utilizar las herramientas más adecuadas en cada situación. La escalera reconstructiva siempre será la base de nuestro actuar como cirujanos plásticos y reconstructivos.

\section{REFERENCIAS}

1. Turner AJ, Parkhouse N. Revisiting the reconstructive ladder. Plast Reconstr Surg 2006; 118 (1): 267-268.

2. Mascaró P. Reconstrucción de tejidos blandos de la extremidad superior. Rev Med Clin Condes 2016; 27 (1): 42-53.

3. Simman R. Wound closure and the reconstructive ladder in plastic surgery. J Am Col Certif Wound Spec 2009; 1: 6-11.

4. Villaverde-Doménech ME, Simón-Sanz E, DelgadoRuíz T, Pérez-Ramos L, Safont-Albert J. El reto de las transferencias de colgajos libres en pacientes quemados ¿̇uál es el mejor momento para la cirugía? Cir Plast Iberolatinoam 2015; 41: 117-126.

5. Baumeister S, Köller M, Dragu A, Germann G, Sauerbier M. Principles of microvascular reconstruction in burn and electrical burn injuries. Burns 2005; 31 (1): 92-98.

6. Ofer N, Baumeister S, Megerle K, Germann G, Sauerbier M. Current concepts of microvascular reconstruction for limb salvage in electrical burn injuries. J Plast Reconstr Aesthet Surg 2007; 60 (7): 724-730.

7. Malard O, Corre P, Jégoux F, Durand N, Dréno B, Beauvillain C et al. Surgical repair of labial defect. Eur Ann Otorhinolaryngol Head Neck Dis 2010; 127 (2): 49-62.

8. López F, Vásquez O, Ocampo J. Reconstrucción de labio: conceptos actuales. Dermatología CMQ 2015; 13 (1): 24-32.

Correspondencia:

Dr. Patricio Canepa Fernández

Salvador Díaz Mirón s/n, Santo Tomás, Alcaldía Miguel Hidalgo,

11340, México, Ciudad de México.

E-mail: drpatriciocanepa@gmaill.com 